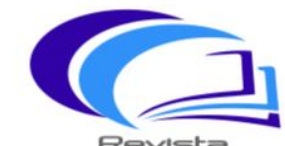

Reviste

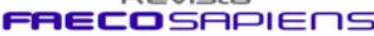

Recibido: 13/04/19 Aceptado: 20/06/19 pp. 01-20 Indexada

\title{
LA FLEXIBILIDAD LABORAL EN LA BANCA PANAMEÑA, FUSIONES Y ADQUISICIONES
}

Flexibility work panamanian bank, fusions and acquisitions

\author{
Roldán Adames Aparicio \\ Profesor Regular, Departamento de Recurso Humanos, Facultad de Administración de Empresas y Contabilidad, \\ Universidad de Panamá \\ roldanadames@cableonda.net
}

\begin{abstract}
RESUMEN
El presente estudio analiza las diversas formas de crecimiento económico que ha experimentado Panamá en las últimas décadas, razón por la cual ha permitido tener un notable crecimiento y fortalecimiento en sus indicadores económicos. No obstante, la realidad del sector bancario ha generado procesos de fusiones, adquisiciones, incorporaciones lo que ha planteado ciertas ventajas y desventajas, sobre todo para el profesional, porque se traduce en desempleo o nuevas formas de organización del trabajo como lo es, la flexibilidad laboral. El estudio se plantea bajo las premisas del análisis discursivo, interpretación y análisis, de la situación actual del sector bancario panameño y las posibles repercusiones en el bienestar familiar, personal y profesional del componente humano dentro de las propias instituciones.
\end{abstract}

Palabras Claves: Flexibilidad Laboral, Fusión, Adquisición, Sector Bancario.

\begin{abstract}
The present study analyzes the different forms of economic growth that Panama has experienced in the last decades, which is why it has allowed a remarkable growth and strengthening in its economic indicators. However, the reality of the banking sector has generated processes of mergers, acquisitions, incorporations which have posed certain advantages and disadvantages, especially for the professional, because it translates into unemployment or new forms of work organization such as flexibility labor. The study is presented under the premises of the discursive analysis, interpretation and analysis of the current situation of the Panamanian banking sector and the possible repercussions on the family, personal and professional well-being of the human component within the institutions themselves.
\end{abstract}

Keywords: Labor Flexibility, Fusion, Acquisition, Banking Sector. 
REVISTA FAECO SAPIENS

ISSN L 2644-3821

Acceso Abierto. Disponible en: https://revistas.up.ac.pa/index.php/faeco_sapiens

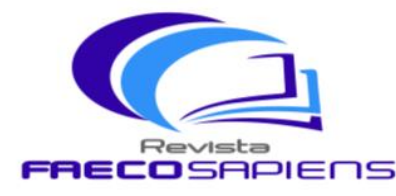

Revista ค月EСロS月คIEחS
शाT Volumen 3 - Número 1

Enero-Junio 2020

Recibido: 13/04/19 Aceptado: 20/06/19 pp. 01-20 Indexada

\section{INTRODUCCIÓN}

Panamá es un país con una economía estable y sólida, que, en comparación con los procesos económicos y sociales del continente y el Caribe, apunta a un crecimiento importante dentro de toda la región. Sobre estas líneas, el Informe de la CEPAL (2018) apunta que nuestro país tendrá para el año 2019, un crecimiento de 5,6\%, y que en el conjunto de América Latina y el Caribe, tendrá un crecimiento 1,7\% con el respecto al año anterior. Por lo que se infiere, que nuestro país cuenta con índices atractivos respecto a la tasa de empleo, el gasto público y las finanzas estatales, que lo ubica en un crecimiento económico positivo los cuales están sujeto a las evaluaciones de indicadores económicos internacionales.

Se aduce, por tanto, que el presente trabajo y bajo el punto de vista de un análisis lógico dialectico, se pretende, desde la rama lógica formal, una interpretación de las ciencias jurídicas, el impacto en los derechos humanos como consecuencia de la flexibilidad laboral referida, específicamente en el periodo de mayor crecimiento, 2009-2014, donde el derecho del trabajo como parte del derecho social, tuvo un álgido debate en atención a las reformas y cambios que se planteaban desde el Estado panameño, y en donde la flexibilidad laboral, como fenómeno económico global tuvo un mayor impacto en la vida de los panameños. Por tanto, es importante poner de manifiesto la tesis de que el crecimiento económico versus la garantía de los derechos básicos de los ciudadanos, como el derecho social, realiza un balance critico de supuestos doctrinales que afirman que la flexibilidad laboral supone un mal menor en comparación de los beneficios que obtienen los ciudadanos a mediano y largo plazo.

Por todo lo antes expuesto, el enfoque del presente trabajo está centrado a exponer las diversas situaciones de índole jurídico y social que tienen como desenlace, dentro de la etapa de fortalecimiento de nuestra económica, un valor contrastante 
REVISTA FAECO SAPIENS

ISSN L 2644-3821

Acceso Abierto. Disponible en https://revistas.up.ac.pa/index.php/faeco_sapiens
Volumen 3 - Número 1

Enero-Junio 2020

Recibido: 13/04/19 Aceptado: 20/06/19 pp. 01-20

Indexada

que evidencia la desproporción de supuestos beneficios que afectan, no solo los derechos económicos y sociales de los panameños, sino que llegan a darle un giro a el espíritu de nuestra constitución política, debilitando cada vez más nuestro ordenamiento jurídico, por tanto, el producto del presente trabajo deberá sentar las bases y criterios que sustenten la búsqueda en la evolución de los conceptos referidos al denominado estado de derecho (liberal), dando paso al estado social de derecho, en el cual se prioriza los derechos individuales y colectivos de cada uno de los ciudadanos, por cuanto que nuestro norte, bajo la óptica critica, de las propias consecuencias del llamado "boom económico", en la referida calidad de vida y bienestar de los panameños y por consiguiente de los propios derechos humanos de los trabajadores.

\section{FLEXIBILIDAD LABORAL}

Las transformaciones globales, y concretamente en el mundo empresarial, han tenido su propio origen en la forma que han ido evolucionando diversos aspectos de la sociedad, las familias, las personas, así como los cambios generados en las propias empresas, razón por la cual se ha tornado imperante que las empresas tengan la necesidad de flexibilizar, es decir la necesidad de cambio en todos los factores que circundan la gestión y operación empresarial. Hay que mencionar que una empresa flexible persigue utilizar lo mejor posible los factores disponibles para adaptarse a los cambios inmediatos y facilitar, a largo plazo, su adaptación al entorno competitivo para lograr un desarrollo organizativo permanente

Con respecto, al análisis de la flexibilidad laboral, Aktouf citado por López Arellano et al. (2015) manifiestan que la flexibilidad laboral se presenta como un fenómeno que tiene orígenes empresariales con profundas y complejas implicaciones sociales sin olvidar que ha contribuido también a generar cambios a nivel tecnológico, jurídico y económico, continua manifestando el autor que el estudio de la flexibilidad laboral 
REVISTA FAECO SAPIENS

ISSN L 2644-3821

Acceso Abierto. Disponible en https://revistas.up.ac.pa/index.php/faeco_sapiens

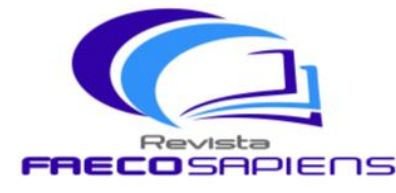

Revista
गाT Volumen 3 - Número 1

Enero-Junio 2020

Recibido: 13/04/19 Aceptado: 20/06/19 pp. 01-20 Indexada

servirá para explicar una práctica empresarial dentro de un contexto socioeconómico particular que vivimos actualmente. Sin embargo, para Rimbau Gilabert y Triadó (2006) aduce que la flexibilidad laboral está referida a la adecuación eficiente de los recursos humanos disponibles y de la organización del trabajo a las variaciones de la demanda de productos y servicios, así como a la diversificación de productos. La dirección de recursos humanos es la responsable por excelencia de desarrollar esta forma de flexibilidad en su empresa. Manifiestan los autores que la flexibilidad laboral debe definirse como la capacidad de adaptación o respuesta que posee la organización en su conjunto ante cambios actuales o previstos del entorno, conseguida a partir de una actitud estratégica, un sistema productivo, una estructura organizativa y una fuerza de trabajo flexibles.

Por otro lado, Fernández Rocha (2014) manifiesta que la flexibilidad laboral debe entenderse como una actitud crítica permanente sobre la regulación de la relación del trabajo y de la misma prestación del servicio, con el fin de adaptarlas a las siempre cambiantes necesidades y exigencias del sistema productivo que se expresa en normas jurídicas y genera individualización de la relación del trabajo. Con ello el autor, aduce que la flexibilización no busca la desmejora de las condiciones de trabajo lo que se pretende es de alguna forma es adecuar la legislación actual y el servicio a los cambios que se viven, sin embargo, esa adaptación, hoy por hoy, supone la desmejora de las condiciones en las que estaban los trabajadores, por lo que se pretende de alguna forma decir que bajo ciertas circunstancias, sería posible que la misma flexibilización incremente o mejore las condiciones de trabajo. Sin embargo, para Raso citado por Mazloum y Isea (2017) infiere que la flexibilidad laboral debe comprenderse como la tendencia generalizada de modificar por vía autónoma o heterónoma las reglas jurídicas de la prestación del trabajo, con la finalidad de ajustar el factor trabajo de las nuevas exigencias del sistema de producción. Por otro lado, Hoyos (1987) opina que la flexibilización laboral radica en la posibilidad de la empresa de contar con mecanismos jurídicos 


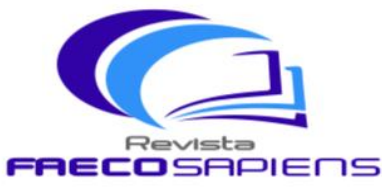

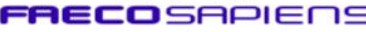

Volumen 3 - Número 1

Enero-Junio 2020

Recibido: 13/04/19 Aceptado: 20/06/19 pp. 01-20

Indexada

que le permitan ajustar su producción, empleo y condiciones de trabajo ante las fluctuaciones rápidas y continuas del sistema económico (demanda efectiva y diversificación de la misma, tasa de cambio, intereses bancarios, competencia internacional), las innovaciones tecnológicas y otros factores que demandan ajustes con rapidez, por lo que ambos conceptos definidos por los autores se puede deducir que la flexibilización laboral consiste en un conjunto de mecanismos de optimización de los recursos humanos, económicos, tecnológicos y físicos de la empresa, con tendencia a profundizarse y mantenerse en el tiempo, cuyo objetivo fundamental es la fácil adaptabilidad de las relaciones laborales a las exigencias económicas del mercado tanto interno como externo, dentro del marco jurídico ${ }^{1}$.

Dado que vivimos en una época en la que combinar el entorno laboral con el ámbito personal es cada vez más complicado, de hecho, es uno de los temas que más debates generan en el día a día. Todo sabemos que el trabajo no solo es necesario para sentirnos realizados personal y profesionalmente, sino también es necesario como una medida de sustento para hacer frente a los gastos que generan tener una familia, aficiones, etc., por lo que es el trabajo se convierte en algo imprescindible en el terreno personal. Con respecto a lo anterior expuesto, que hacerse los siguientes cuestionamientos, ¿Qué ocurre cuando nuestro entorno laboral invade nuestro espacio personal? o, ¿Qué pasa cuando nuestro trabajo nos impide disfrutar de nuestra familia, amigos aficiones, etc.?, para comprender mejor estos cuestionamiento Quiroz (2017) aduce que la importancia en todos los ámbitos de la

\footnotetext{
1 Países como Francia, España, Italia, Inglaterra, Alemania, Suecia han modificado sus normas laborales con la finalidad de dar cabida a la flexibilización; razón por la cual, y de hecho la polémica del tema tiene sus principios en Europa en los años 1974-76, por un lado, y por el otro, América Latina, con efectos diferentes al europeo a principios de la década de los años 80 , por lo que se suscitaron procesos encadenados a través de las reformas de las leyes laborales adaptándolas a la fuentes flexibilizadoras, que propinaban una favorecimiento en el empleo, la inversión y la competitividad internacional de las diversas empresas, basándose en argumentos válidos sobre el decaído y deprimido continente. Es por ello, que países como Argentina, Brasil, Chile, Colombia, Ecuador, El Salvador, México y Paraguay, dieron cabida a la estructuración de normas que han flexibilizado las relaciones laborales.
} 
REVISTA FAECO SAPIENS

ISSN L 2644-3821

Acceso Abierto. Disponible en https://revistas.up.ac.pa/index.php/faeco_sapiens

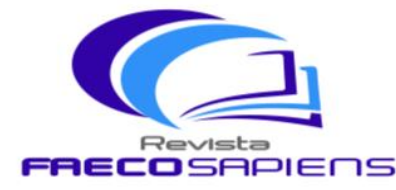

Revista
शाT Volumen 3 - Número 1 DRJI

Enero-Junio 2020

Recibido: 13/04/19 Aceptado: 20/06/19 pp. 01-20 Indexada

vida es buscar el equilibrio, y por supuesto, no se puede menoscabar el entorno laboral, razón por la cual debemos buscar un equilibrio tanto en el ámbito laboral como en el ámbito personal, de esta forma se benefician tanto las empresas, a las que nada le vale tener colaboradores desmotivado al acumular largas jornadas de trabajo, así como, de nada sirve que los empleados trabajen más de lo normal si después no van a tener tiempo para disfrutar su vida familiar y personal. Se aduce, por tanto, que cuando hablamos de flexibilidad laboral es preciso referirse a la racionalización del trabajo, a la productividad, a encontrar el equilibrio entre los intereses de la persona y los de la empresa. $Y$ es que todas estas cualidades tienen un mismo fin, conciliar la vida personal con la profesional. Cabe señalar que la flexibilidad laboral surge, pues, y se convierte en algo necesario a emprender en todas las empresas, convirtiéndose en una herramienta clave para mitigar el problema de la escasez de tiempo en los colaboradores, que a su vez, repercutiría muy favorablemente en la producción de la empresa

En los últimos tiempos, la organización de las empresas como entidad de producción de bienes y servicios debe ser considerada como entidades flexibles; para ello se necesita mano de obra flexible, para ello, es preciso considerar las empresas flexibles bajos los principios de la flexibilidad empresarial. La flexibilidad empresarial, por tanto, nace como respuesta a las necesidades de las empresas, de hacer frente a los constantes cambios, que son demandados por los clientes y los consumidores, es por ello, que las empresas deben de ser flexibles, ante dichos cambios en los gustos de los consumidores, ya que existe una gran incertidumbre en el mercado de trabajo.

A este respecto Albizu (1997) manifiesta que la flexibilidad empresarial, es la capacidad que debe de tener una organización, para poder adaptarse a los continuos cambios, (ya sean cambios económicos, tecnológicos, sociales o culturales), que puedan afectar al contexto interno y externo de la organización. 
REVISTA FAECO SAPIENS

ISSN L 2644-3821

Acceso Abierto. Disponible en https://revistas.up.ac.pa/index.php/faeco_sapiens

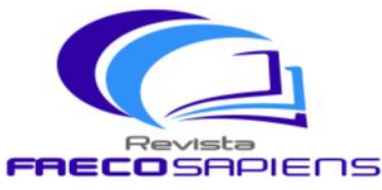

Revista
ताT Volumen 3 - Número 1

Enero-Junio 2020

Recibido: 13/04/19 Aceptado: 20/06/19 pp. 01-20 Indexada

Esta capacidad, de la cual puede disponer cualquier organización, puede beneficiar a la empresa, en el sentido de mantenimiento o mejora de la posición competitiva, dentro del mercado laboral. Así pues, la flexibilidad es lo contrario a rigidez; porque lo que caracterizo a las empresas diseñadas para obtener un solo producto y en grandes volúmenes, con un sistema técnico muy especializado, hoy por hoy, esta concepción no respondía a las exigencias del mercado, el cual pedía diversificación de productos y servicios, y en pequeñas cantidades.

Lo manifestado hasta aquí, y expuesto por los estudiosos de la temática abordada, la flexibilidad laboral implica, para unos adecuar el mercado laboral a las exigencias de las necesidades que demandan los clientes y consumidores, a través de normas que han desregularizado el contrato por tiempo indefinido en compromisos más a corto plazo, implicando mejor calidad de vida laboral y personal, mientras que para otros la enfatizan como una precariedad que dejan al trabajador totalmente indefensos.

\section{FLEXIBILIDAD LABORAL EN PANAMÁ}

A fines de 1976, luego del impacto de la crisis del petróleo y la crisis económica que se vivió en Panamá, comienza una etapa de flexibilización de la legislación laboral. El inicio de un proceso flexibilizador dentro del mercado laboral en Panamá coincide igualmente con la etapa final de las negociaciones de un nuevo Tratado del Canal con los Estados Unidos de América. Destacan legislación que repercuten en la relación laboral panameña, a saber:

- La ley 95 de 31 de diciembre de 1976, esta ley introdujo reformas flexibilizadoras al Código de Trabajo, que afectaron precisamente parte de las principales áreas que caracterizaron la diferenciación del mismo respecto de la legislación anterior. En este sentido, con una fórmula ambigua e imprecisa, 
REVISTA FAECO SAPIENS

ISSN L 2644-3821

Acceso Abierto. Disponible en: https://revistas.up.ac.pa/index.php/faeco_sapiens

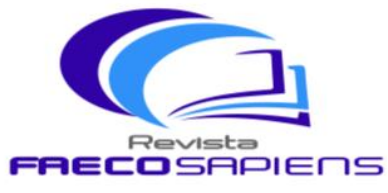

Revista

\section{ताT Volumen 3 - Número 1 DRJI Enero-Junio 2020}

Recibido: 13/04/19 Aceptado: 20/06/19 pp. 01-20 Indexada

eliminó el régimen de estabilidad en el empleo, al trasladar al empleador la opción entre reintegro e indemnización; introdujo el silencio administrativo positivo, a favor del empleador, en las autorizaciones de despido por causas económicas; limitó la obligatoriedad de celebrar la convención colectiva de trabajo, al establecer que la empresa no estaba obligada a negociar si se afectaba su rentabilidad económica.

- La ley 8 de 30 de abril de1981, también de tendencia flexibilizadora, intentó atemperar los efectos de la ley 95 de 1976.

- La ley 1 de 17 de marzo de 1986, esta ley fue el producto de demandas de las instituciones financieras de crédito y representa una clara orientación desreguladora.

- Otras normas aprobadas en el período, en líneas generales, este período se caracteriza por representar una etapa de replanteamiento de la legislación laboral, en términos flexibilizadores dirigidos a una disminución de la protección laboral y a una reducción de los costos laborales. Entre la normativa adoptada en este período, podemos mencionar:

a. Leyes sobre aumentos generales de salario (10 de 1979 y 13 de 1981).

b. Ley 10 de 4 de junio de 1981, adiciona dos días de fiesta nacional (10 y 28 de noviembre).

c. Ley 5 de 2 de marzo de 1982, relativa al trabajo en la construcción en obras especializadas o de interés nacional.

d. Ley $\mathrm{N}^{\circ} 13$ de 30 de abril de 1982, sobre el trabajo en la construcción.

e. Ley $\mathrm{N}^{\circ} 16$ de 4 de junio de 1982, por medio de la cual se toman medidas sobre salarios en las empresas constructoras, (Weller, 2009).

f. Ley 44 de agosto de 1995, relativa a productividad, flexibilidad y beneficios fiscales.

La evolución en la naturaleza de la fuerza del trabajo, en Panamá, pasa por cambios profundos en el comportamiento de las nuevas generaciones que se integran a la 
REVISTA FAECO SAPIENS

ISSN L 2644-3821

Acceso Abierto. Disponible en: https://revistas.up.ac.pa/index.php/faeco_sapiens

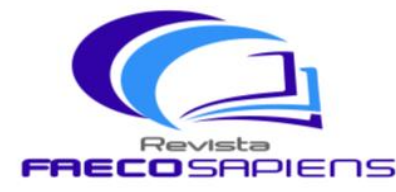

Reviste
शTा Volumen 3 - Número 1

Recibido: 13/04/19 Aceptado: 20/06/19 pp. 01-20 Indexada

vida laboral (Martin, 2016). Los colaboradores que disfrutan de una mayor flexibilidad laboral tienen menos ausencias y muestran un mayor compromiso con su trabajo. Contínua manifestando que las empresas pueden aprovechar de mejor manera los hábitos de las nuevas generaciones, como los nómadas digitales, al crear entornos culturales de alta inclusión, alto compromiso, un efectivo balance entre la vida personal y el trabajo, así como una relación de coaching y aprendizaje continuo con sus líderes, que facilitan las tecnologías de colaboración para habilitar el trabajo flexible o remoto. En cuanto a políticas, las empresas deben orientarse a objetivos, basar la cultura en un ambiente de confianza, una mentalidad de crecimiento y aprendizaje, responsabilidad y empoderamiento, impulsar la diversidad e inclusión, reconocer las conductas adecuadas y, lo más importante, liderar con el ejemplo.

\section{FUSIONES Y ADQUISICIONES EN LA BANCA PANAMEÑA}

El sistema bancario de Panamá ha experimentado en las últimas décadas un notable crecimiento, el fortalecimiento de sus indicadores y en el sistema bancario. Así pues, en los últimos 20 años se han registrado fusiones, transformaciones e incorporaciones en dichos sistema que lo han convertido en uno de los más importantes centros financieros de América Latina. Esto último también se ha visto favorecido por las facilidades legales que ofrece Panamá para instalar empresas, por la promoción de las inversiones que realiza el Estado, por la ubicación geográfica del país en relación a la región y, sobre todo, por la relativa estabilidad económica y política que ofrece el país.

Brealey y Myers (1993) enfatizan que las fusiones vienen en flujos que datan desde comienzos de siglo y el segundo en los años veinte y a las que se les atribuye diferentes fuerzas impulsoras. Sobre este aspecto, Kenneth (1985) subraya cuatro fuentes de flujos de fusiones, entre las que destacan: 


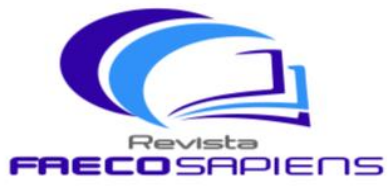

Revista

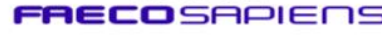

Volumen 3 - Número 1

Enero-Junio 2020

Recibido: 13/04/19 Aceptado: 20/06/19 pp. 01-20

Indexada

- Integración Horizontal, siendo la fuerza impulsora la necesidad de las empresas de aumentar su capacidad de fabricación, beneficiándose con la generación de economías de escala.

- Abarca aproximadamente desde 1920 hasta 1929, recibió el nombre de Integración Vertical, siendo el objetivo la reducción de los costos operativos para mantener los márgenes de beneficios, a través del control sobre toda la cadena productiva.

- Las empresas pretendían liberarse de la reglamentación anti-trusts y estabilizar su rendimiento financiero. Esto último lo lograban comprando empresas con capacidad para contraer mayores deudas y proporcionar suficiente efectivo para llevar adelante nuevas adquisiciones. Este flujo se le denomino Conglomeración.

- caracterizada por las Megafusiones, a causa de la realización de fusiones que involucran cifras de gran magnitud.

Las fusiones bancarias son operaciones por lo que las entidades de créditos independientes se disuelven y ceden sus activos y pasivos a otra sociedad nueva 0 existente. El objetivo es conseguir un banco más eficiente, de mayor categoría y apto para competir con las grandes entidades del mercado. Aunque las razones por las fusiones bancarias pueden ser varias, normalmente son dos:

- Para mejorar la posibilidad de crecimiento de bancos pequeños.

- Superar la crisis y evitar el cierre de las entidades con más problemas de liquidez.

Desde el punto de vista legal, la fusión es la unión jurídica de dos o más sociedades que se compenetran recíprocamente para que una sola de ellas, como organización jurídica unitaria, sustituya la pluralidad de entes de derecho. 


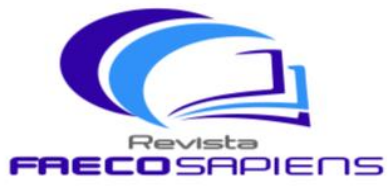

Revista

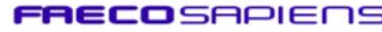

Volumen 3 - Número 1 Enero-Junio 2020

Recibido: 13/04/19 Aceptado: 20/06/19 pp. 01-20 Indexada

Bajo el enfoque financiero, en la práctica, la fusión se ha convertido en una operación que puede llevarse a cabo a través de:

- Fusión por absorción, que ocurre cuando una sociedad absorbe o incorpora los asociados, el patrimonio, y la totalidad de derechos y obligaciones de la(s) sociedad(es) absorbida(s).

- Fusión por creación, que se realiza cuando sociedades en fusión se disuelven (desaparecen) y funden todos sus elementos patrimoniales en una nueva compañía, para proseguir en derechos y obligaciones.

Por lo general, las fusiones bancarias son producidas por la búsqueda de economías de sucesión o de ventajas monopólicas; en el primer caso, son una expresión del proceso de integración horizontal o vertical entre empresas independientes, que buscan una mayor eficiencia en sus procesos productivos; y en el segundo caso, puede considerarse como una forma de control en los mercados. En esencia los procesos de fusión, buscan mejorar la eficiencia y competitividad de los bancos a través de economías de escala, para fortalecer su posición dentro o fuera del Centro Financiero de Panamá.

La Superintendencia de Bancos de Panamá manifiesta que las fusiones y adquisiciones bancarias surgen como efecto de la creación de una nueva razón social, Tabla 1, resultante de la disolución de dos o más entidades o por la fusión, que implique el traspaso del $100 \%$ sus respetivos capitales de la entidad con la nueva razón social, la cual también adquirirá la totalidad de los derechos y obligaciones de las instituciones fusionadas. 
REVISTA FAECO SAPIENS

ISSN L 2644-3821

Acceso Abierto. Disponible en:

https://revistas.up.ac.pa/index.php/faeco_sapiens

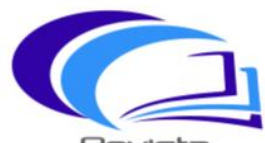

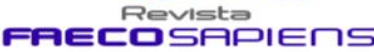

Volumen 3 - Número 1

Enero-Junio 2020

Recibido: 13/04/19 Aceptado: 20/06/19 pp. 01-20 Indexada

Tabla 1. Fusiones Bancarias en Panamá 2000-2013

\begin{tabular}{|c|c|c|c|}
\hline Año & Entidad & Proceso & Sobreviviente \\
\hline \multirow[t]{8}{*}{2000} & Banco Bilbao Vizcaya (Panamá), S.A. & Fusión por absorción & Banco Exterior, S. A. \\
\hline & Banco Comercial de Panamá & Fusión por absorción & Banco General, S.A. \\
\hline & Primer Banco de Ahorro, S.A. & Adquisición & Banco del Istmo, S.A. \\
\hline & The Chase Manhattan Bank & Traspaso & HSBC Bank USA \\
\hline & Primer Grupo Nacional, S.A. & Fusión & Banco del Istmo, S.A. \\
\hline & National Baltic Investment, S.A. & Fusión & Banco General, S.A. \\
\hline & 7th Ocean Marvel, Inc. & Fusión & Banco Internacional de Panamá, S.A. \\
\hline & Banco Bilbao Vizcaya (Panamá), S.A. & Fusión por absorción & Banco Exterior, S. A. \\
\hline \multirow[t]{8}{*}{2001} & Norfolk and Hampton Bank & Adquisición & Banco Continental de Panamá, S.A. \\
\hline & Dresdner Bank AG & Traspaso & Allianz Bank Beteilung GmbH \\
\hline & ABN AMRO Bank, N.V. (Panamá) & Traspaso & Banco Mercantil del Istmo, S. A. \\
\hline & Primer Banco del Istmo, S.A. & Fusión por absorción & Primer Banco de Ahorros, S.A. \\
\hline & Bank \& Trust, Ltd. & Adquisición & Popular Bank Cayman Limited. \\
\hline & Financial Holding Company, S.A. & Fusión por absorción & Banco Continental de Panamá, S.A. \\
\hline & Bancredit Cayman Limited & Adquisición & Bancrédito (Panamá), S.A. \\
\hline & Norfolk and Hampton Bank & Adquisición & Banco Continental de Panamá, S.A. \\
\hline \multirow[t]{8}{*}{2002} & Bipan Holding Corp & Fusión & Grupo Continental, S.A. \\
\hline & Inversiones Latinoamericanas, S.A. & Fusión & Primer Banco del Istmo, S.A. \\
\hline & Banco Internacional de Panamá, S.A. & Fusión por absorción & Banco Continental de Panamá, S.A. \\
\hline & Banco de Latinoamérica, S.A. & Fusión & Primer Banco del Istmo, S.A. \\
\hline & BANCO BCT (PANAMA), S.A. & Fusión & BCT BANK INTERNATIONAL, S.A. \\
\hline & Discount Bank. & Fusión & Bancaire Privee \\
\hline & Bipan Holding Corp & Fusión & Grupo Continental, S.A. \\
\hline & Inversiones Latinoamericanas, S.A. & Fusión & Primer Banco del Istmo, S.A. \\
\hline \multirow[t]{4}{*}{$2003-2004$} & Banco Panamericano, S.A., Ancón Holding Panamá, S.A. & Fusión por absorción & Banco Cuscatlán de Panamá, S.A. \\
\hline & Grupo Wall Street Securities, S.A. y a Wall Street Bank, S.A. & Fusión por absorción & Banco Continental de Panamá, S.A. \\
\hline & $\begin{array}{l}\text { Bienes Raíces Portobello, S.A., Inmobiliaria de América Latina, } \\
\text { S.A., y Bienes y Valores Monterrey, S.A. }\end{array}$ & Fusión por absorción & Primer Banco del Istmo, S.A. \\
\hline & Banco Mercantil del Istmo, S.A. & Fusión por absorción & Primer Banco del Istmo, S.A. \\
\hline \multirow[t]{7}{*}{ 2005-2006 } & Banco Atlántico (Panamá), S.A. & Fusión por absorción & Banco Atlántico (Panamá), S.A. \\
\hline & Naka Investment Corporation & Fusión por absorción & Naka Investment Corporation \\
\hline & Credicorp Financial Inc., y Financiera La Suerte, S.A. & Fusión con & $\begin{array}{l}\text { Credicorp Financial Inc., y Financiera La } \\
\text { Suerte, S.A. }\end{array}$ \\
\hline & Atlántico Leasing, S.A. y Atlántico Servicios, S.A. & Fusión por absorción & $\begin{array}{l}\text { Atlántico Leasing, S.A. y Atlántico } \\
\text { Servicios, S.A. }\end{array}$ \\
\hline & BICSA Corporación Financiera, S.A. & Fusión con & $\begin{array}{l}\text { BICSA CORPORACIÓN FINANCIERA, } \\
\text { S.A. }\end{array}$ \\
\hline & General Investment Securities, Inc. & Fusión por absorción & General Investment Securities, Inc. \\
\hline & Banco Atlántico (Panamá), S.A. & Fusión por absorción & Banco Atlántico (Panamá), S.A. \\
\hline \multirow[t]{13}{*}{$2007-2008$} & Banex International Bank Corp. & Fusión por absorción & GTC Bank Inc. \\
\hline & $\begin{array}{l}\text { Leesport Finance, Inc. Dramen International, Inc. Dontobay } \\
\text { Holding, Inc. y Drabox International, S.A. }\end{array}$ & Fusión por absorción & Global Bank Corporation, Inc. \\
\hline & Inversiones Far West, S.A. & Fusión por absorción & Banco General, S.A. \\
\hline & Servicios Centenarios de Panamá, S.A. & Fusión & Banco Panamá. S.A. \\
\hline & Primer Banco del Istmo, S.A. & Fusión & HSBC Bank (Panamá) S.A. \\
\hline & Grupo BANISTMO, S.A., & Fusión por absorción & HSBC Bank (Panamá) S.A. \\
\hline & BanAgricola, S.A. & Adquisición & Bancolombia (Panamá), S.A. \\
\hline & PROMERICA, S.A. & Fusión por absorción & Saint Georges Bank \& Company, Inc. \\
\hline & Banco Aleman, Platina, S.A. & Fusión por absorción & Banco Continental de Panamá, S.A. \\
\hline & Suleasing Internacional, S.A. & Fusión & Bancolombia (Panamá) S.A. \\
\hline & $\begin{array}{l}\text { Corporación Financiera Miravalles, S.A., Recaudadora Costa } \\
\text { Rica, S.A. (RECSA) }\end{array}$ & Fusión por absorción & Banco BAC San José, S.A. \\
\hline & Banco Continental de Panamá, S.A. & Fusión por absorción & Banco General, S.A. \\
\hline & GRANBANCO, S.A. & Fusión por absorción & Banco Davivienda, S.A. \\
\hline \multirow[t]{3}{*}{$2010-2011$} & Administradora Ancón, S.A. Factoring Cuscatlan & Fusión por absorción & Banco Citibank (Panamá), S.A. \\
\hline & Uni B \& T de Panamá, S.A. & Fusión por absorción & Uni Bank \& Trust Inc. \\
\hline & BAC International Bank Inc.. & Traspaso & Leasing Bogota, S.A. Panamá \\
\hline \multirow[t]{3}{*}{$2012-2013$} & Banco Trasatlántico, S.A. & Fusión por absorción & Balboa Bank \& Trust, Corp. \\
\hline & Inmobiliaria Universal 50, S.A. & Fusión por absorción & Banco Universal, S.A. \\
\hline & Hsbc Bank (Panamá), S.A. & Adquisición & Bancolombia, S.A. \\
\hline
\end{tabular}

Fuente: Elaboración propia a partir de la información obtenida de la Superintendencia de Bancos de Panamá. 


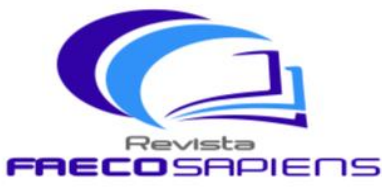

La absorción de una o más instituciones por otra, lo cual conllevará la desaparición de la (s) entidad (es) absorbida (s) y el traspaso de la universalidad de su(s) capital (es), activos y pasivos a la entidad absorbente. La compra de la totalidad de las acciones de la entidad absorbida por parte de la entidad absorbente. Como consecuencia de esta operación, y fuego de la comprobación del hecho mediante hacia notarial y de haberse cumplido con las formalidades establecidas en el Código de Comercio y en las disposiciones legales vigentes, los activos y pasivos de la entidad absorbida se integrará al capital de la entidad absorbente quedando la primera disuelta y liquidada de pleno derecho.

Panamá cuenta hoy con 91 bancos instalados en el país a pesar de los múltiples procesos de fusión y adquisición, Fig. 1 y Fig. 2, que se han dado en los últimos meses a nivel bancario. La historia ha demostrado que el sistema bancario panameño está en su mejor momento. Después de haber superado un sinnúmero de escollos, las experiencias ganadas le han permitido aprender y modificar cada uno de los elementos necesarios para mantenerse a la vanguardia exigida en el momento, conservando su solvencia moral, económica y estabilidad, atesorando lo que mejor sabe hacer, prestar un excelente servicio para Panamá y el mundo.

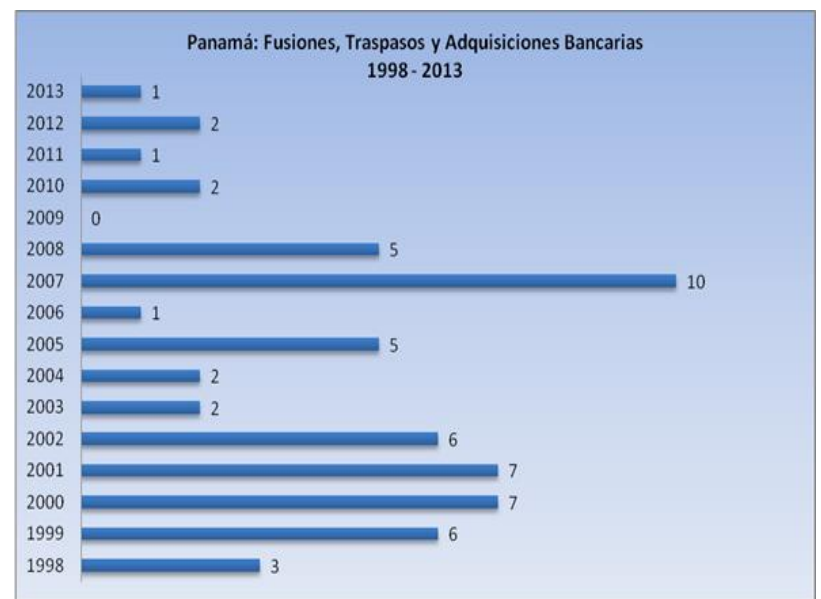

Figura 1. Adquisiciones y Traspasos $1998-2013$

Fuente: https://www.monografias.com/trabajos97/fusiones-bancarias-panama-2013/fusiones-bancarias-panama-2013.shtml 


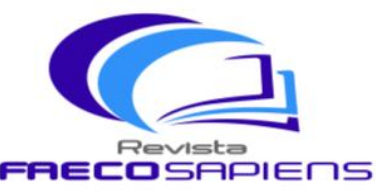

\section{Por Tipo de Acción} $1998-2013$

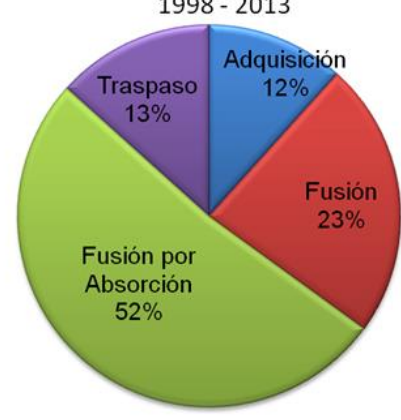

Figura 2. Tipo de Acciones de las Adquisiciones y Traspasos 1998 - 2013

Fuente: https://www.monografias.com/trabajos97/fusiones-bancarias-panama-2013/fusiones-bancarias-panama-2013.shtml

\section{FUSIONES Y ADQUISICIONES BANCARIAS EN SECTOR LABORAL}

El gran flujo de fusiones y adquisiciones bancarias que en los últimos años se ha presentado está estructurando los servicios bancarios y financieros, por lo que según la Organización Internacional del Trabajo (OIT), se está agravando la disminución del empleo global en un sector tradicionalmente caracterizado por un empleo estable e incluso de por vida. Según el informe de la OIT (2001), el derrumbamiento de las expectativas de las fusiones y adquisiciones obedece en gran parte a los inconvenientes de la reducción del personal, que puede redundar en el deterioro del rendimiento de la empresa y en una baja de la moral del empleado. Entre las consecuencias importantes del aumento de la actividad en materia de fusiones para la fuerza del sector financiero que ha logrado sobrevivir al redimensionamiento, se mencionan "la menor seguridad en el empleo, el aumento de la carga de trabajo, la ansiedad y el estrés", que pueden influir negativamente en el rendimiento en un clima de trabajo altamente competitivo.

Como se ha evidenciado lo manifestado por la Organización Internacional del Trabajo, si bien es cierto buena parte de las fusiones y adquisiciones se llevan a cabo en funciones de consideraciones financieras, su éxito depende básicamente de la motivación de los trabajadores que permanecen en sus puestos de trabajo para contribuir al logro de los objetivos de la fusión. Es posible que la elevada 
REVISTA FAECO SAPIENS

ISSN L 2644-3821

Acceso Abierto. Disponible en https://revistas.up.ac.pa/index.php/faeco_sapiens

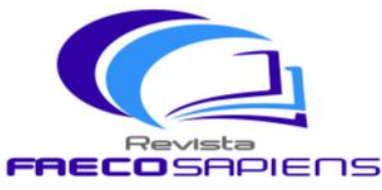

Revista
शTा Volumen 3 - Número 1 DRJI

Enero-Junio 2020

Recibido: 13/04/19 Aceptado: 20/06/19 pp. 01-20 Indexada

proporción de fracasos en las fusiones y adquisiciones esté vinculada al hecho de que con frecuencia el personal es relegado a la variable de costos, en vez de considerarse participantes activos del cambio. Por lo que es necesario distinguir entre, como manifiesta Haspeslagh y Jemison (1991), las adquisiciones de simbióticas, entidades bancarias que primero se mantienen autónomas para luego ser gradualmente interdependientes, por un lado, y por el otro de absorción, ya que en estos casos se producen cambios significativos tanto en la configuración de la organización como en su funcionamiento, por lo tanto, la incertidumbre es mayor, con el subsiguiente ajuste en los recursos humanos. En cambio, en las situaciones de preservación, los cambios son relativamente escasos de manera que éstos afectan principalmente a los niveles directivos más altos, pero el ajuste global en recursos humanos es poco significativo.

Dicho lo anterior, se manifiesta que una característica común de estos procesos es su incidencia sobre el ámbito laboral, tanto en relación a sus posibles efectos a corto plazo sobre el empleo como, fundamentalmente, por el notable impacto que provocan sobre las condiciones de trabajo y relaciones laborales de las empresas implicadas, afectando a múltiples dimensiones: desde el modelo de gestión de la nueva empresa -en el que normalmente suele imponerse la cultura de la empresa dominante en la operación- a la modificación de las condiciones laborales de los trabajadores, o las relaciones con las organizaciones que representan a los mismos. Consecuencias relevantes de estas fusiones y adquisiciones se generan una serie de impactos que repercuten directamente en el empleo del sector y entre los que cabe destacar están:

- Pérdida de identidad.

- Falta de información, comunicación pobre o inconsistente.

- Temor a la pérdida de trabajo.

- Alteración en la carrera profesional.

- Posibilidad de cambio del puesto de trabajo. 


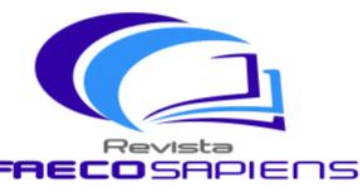

ताI Volumen 3 - Número 1 DRJI

Recibido: 13/04/19 Aceptado: 20/06/19 pp. 01-20 Indexada

- Pérdida o reducción de poder, estatus o prestigio.

- Cambios en las normas y procedimientos, por tanto, aumento en el volumen de trabajo.

- Cambios en compañeros, jefes y subordinados.

- Ambigüedad en las normas, procedimientos y sistemas de información y de recompensa.

- Disminución de la necesidad y devaluación de las viejas habilidades y conocimientos.

- Discriminaciones en base a la cultura.

- A veces se encuentra atrapado porque se ve demasiado joven para retirarse y demasiado mayor para buscar otro trabajo.

- Hay una tendencia a llevar las tensiones de la fusión al entorno familiar.

Por todo lo antes expuesto, cabe señalar que el cambio inducido por una fusión o adquisición significa pasar de un pasado conocido a un futuro, Fig. 3, esencialmente desconocido, ya sea porque en el momento del acuerdo no existen planes detallados o porque están incompletos. El futuro de las entidades bancarias que se fusionan puede estar perfectamente claro para los actores clave, y que están activamente implicados en el proceso, sin embargo, son pocas las personas que en la firma compradora conocen los planes de futuro, mientras que en la firma adquirida puede ser un completo misterio.

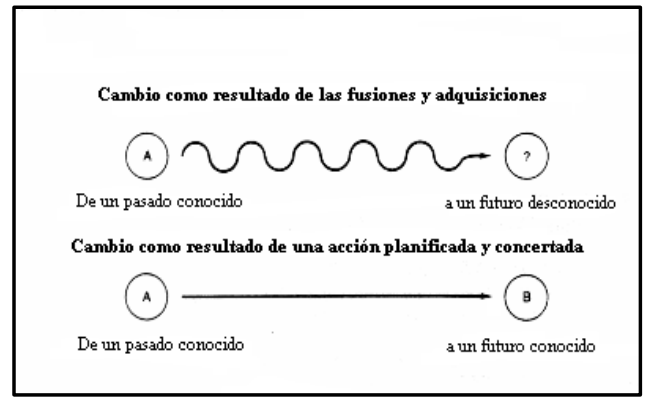

Figura 3. Cambios Generados por los procesos de Fusión y Adquisición Bancaria

Fuente: Escobar, Iveth (s/f). Fusión y Adquisición. Disponible en: https://www.monografias.com/trabajos15/fusionadquis/fusion-adquis.shtml. [En línea]. Fecha de consulta: 28 de junio de 2019. 
REVISTA FAECO SAPIENS

ISSN L 2644-3821

Acceso Abierto. Disponible en: https://revistas.up.ac.pa/index.php/faeco_sapiens

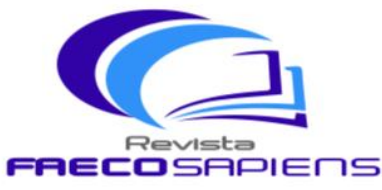

Revista ค月Eடロร月คIEחS
Volumen 3 - Número 1

Enero-Junio 2020

Recibido: 13/04/19 Aceptado: 20/06/19 pp. 01-20 Indexada

De aquí que con estas líneas discursivas, se establece el objetivo que se pretende con presente estudio es el de determinar ¿Cuál ha sido el papel de la flexibilidad laboral en la banca panameña como resultado de las fusiones y adquisiciones que han suscitado en nuestro país en la última década?, de manera que nos permita conocer, mucho más en que consiste la flexibilidad laboral y su implicación en el mercado laboral, como tendencia para enfrentar el neoliberalismo y sus consecuencias en una economía globalizada y en donde no está exenta el centro financiero panameño más puntualmente, la banca panameña, la cual ha sido sometida a las estrategias de fusiones y adquisiciones para ser más competitiva en un mercado disruptivo que para poder enfrentarlo recurren a modalidades para satisfacer las necesidades de clientes y consumidores mediante la flexibilidad del mercado laboral.

\section{METODOLOGÍA}

El presente estudio se estableció bajo los principios de un enfoque cualitativo, no experimental, razón por el cual, el presente estudio se recurrió a fuentes bibliográficas secundarias (Informe de la OIT, de la Superintendencia de Bancos, entre otras) que nos permitió, de alguna manera, establecer desde una perspectiva social un análisis hermenéutico de los elementos que toman postura dentro del mismo, es decir la flexibilidad laboral como resultado de las fusiones y adquisiciones bancarias en nuestro país. Al este respecto, Max Weber (1996) aduce sobre el "entender" de las acciones humanas como conductas subjetivamente significativas, de manera tal que busca establecer y dar sentido a las acciones, hechos o situaciones sociales. Así mismo, Vigo (2002) manifiesta que el propósito que se pretende es la búsqueda de sentido, significado y verdad de las acciones sociales tomando como centro de análisis al individuo. En el caso que nos ocupa en este trabajo, por tanto, es análisis bajo parámetros de interpretación como se ha manifestado la flexibilidad laboral como producto de las fusiones y adquisiciones 


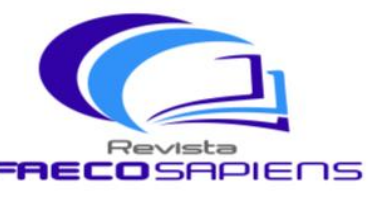

ताI Volumen 3 - Número 1 DRJI Enero-Junio 2020

Recibido: 13/04/19 Aceptado: 20/06/19 pp. 01-20 Indexada

bancarias, y como el individuo dentro del sector ha podido comprender esta situación dentro de su vida personal y familiar.

De igual forma, se consultó con varios a través de relatos personales con varios colaboradores de las entidades bancarias que han estado vinculados y expuestos a los procesos de fusiones y adquisiciones dentro del sector bancario panameño como se desarrolló su experiencia personal y familiar después del proceso dentro de sus entidades bancarias.

\section{RESULTADOS}

Como resultado del presente trabajo, las bases teóricas consultadas han revelado que la flexibilidad laboral como producto de las fusiones y adquisiciones bancarias concretizan una serie de ventajas y desventajas, Tabla 2, tanto para el individuo como para las entidades y que las mismas han influido en su propia vida familiar, personal y profesional o laboral, a saber:

Tabla 2. Ventajas y Desventajas de las Fusiones y Adquisiciones bancarias

\begin{tabular}{|c|c|}
\hline Ventajas & Desventajas \\
\hline $\begin{array}{l}\text { - Mejora la calidad del Servicio Bancario. } \\
\text { - Bajan los costos de transformación. } \\
\text { - Bajan los costos de operación } \\
\text { y producción. } \\
\text { - Solidez y en } \\
\text { el Mercado Financiero. } \\
\text { - Competitividad en el Mercado Financiero. } \\
\text { - Administración más metódica y } \\
\text { fiscalización centralizada. }\end{array}$ & $\begin{array}{l}\text { - } \begin{array}{l}\text { Bajan los Pasivos Laborales (despidos } \\
\text { Masivos). }\end{array} \\
\text { - } \begin{array}{l}\text { Dependiendo como está la economía del } \\
\text { - }\end{array} \text { Creación la fusión será una buena estrategia. } \\
\text { - Posible pánico y confusión en el público. } \\
\text { - La cartera de Crédito en litigio y demorada } \\
\text { a la hora de fisionarse. }\end{array}$ \\
\hline
\end{tabular}

Fuente: Escobar, Iveth (s/f). Fusión y Adquisición. Disponible en: https://www.monografias.com/trabajos15/fusionadquis/fusion-adquis.shtml. [En línea]. Fecha de consulta: 28 de junio de 2019.

Por otro lado, los colaboradores consultados, la mayoría de ellos, coincidieron en 


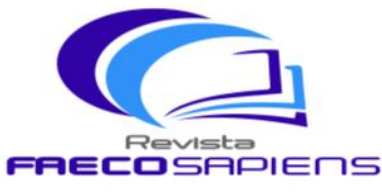

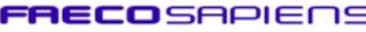

Volumen 3 - Número 1

Enero-Junio 2020

Recibido: 13/04/19 Aceptado: 20/06/19 pp. 01-20 Indexada

que por sus delicadas funciones en la mayoría de las veces trabajaban desde su casa, pero en ocasiones en horas de la madrugada debido a las diferencias horarias de otros países, alegan también que han invertido en tecnología, que, si bien los horarios les han permitido atender otras necesidades de tipo familiar, les es un poco difícil trabajar en solitario. Varios de estos colaboradores han sido producto de las fusiones que han emigrado a esos bancos por sus experiencias, capacitación en le fusiones que han emigrado a esos bancos por sus experiencias, capacitación en el extranjero, formación académica, antes de que sean contratados nuevamente.

A modo de conclusión, si bien se concibe las fusiones y adquisiciones como una estrategia de fortalecimiento económico, pueden generar organizaciones débiles en cuanto a su funcionamiento interno (negociaciones, soluciones de conflicto) y su cultura organizacional, por tanto, es preciso que se establezcan procesos no rígidos, de forma tal que se puede crear empleo formal que garantice el bienestar del individuo a través de un proceso adecuado y flexible en el entorno laboral.

\section{REFERENCIAS BIBLIOGRÁFICAS}

Albizu, Eneka (1997). Flexibilidad laboral y gestión de los recursos humanos. Editorial Ariel. Barcelona, España.

CEPAL - Comisión Económica para América Latina y el Caribe- (2019). Balance Preliminar de las Economías de América Latina y el Caribe, 2018. Publicaciones de las Naciones Unidas. (LC/PUB.2019/1-P). Santiago de Chile, Chile.

Fernández Rocha, Andrés Felipe (2014). La flexibilización Laboral. Disponible en: https://derechopublicom.com/2014/09/la-flexibilizacion-laboral.html. [En línea]. Fecha de Consulta: 24 de junio de 2018.

Haspelagh, P. y Jemison, D. (1991): «Managing acquisitions. Creating value through corporate renewal», The Free Press, New York.

Hoyos, A. (1987). La Flexibilidad del Derecho Laboral Tradicional. Tendencias internacionales recientes. Revista Derecho Laboral. Montevideo, Uruguay.

López Arellano, Hernán E. et Al (2015). Flexibilidad laboral, análisis conceptual y su incidencia en los países latinoamericanos, recientes debates. Disponible en: https://dialnet.unirioja.es/servlet/articulo?codigo=5857395. [En línea]. Fecha de consulta: 28 de julio de 2019

Martin, Enrique (2016). Digitalización y flexibilidad: factores de impacto en el mundo laboral. Disponible en: http://laestrella.com.pa/opinion/columnistas/digitalizacion-flexibilidad-factores- 


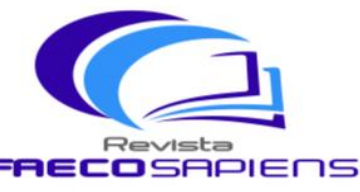

ताI

Volumen 3 - Número 1 DRJI

Enero-Junio 2020

Recibido: 13/04/19 Aceptado: 20/06/19 pp. 01-20 Indexada

impacto-mundo-laboral/23961686. [En línea]. Fecha de consulta: 28 de julio de 2019.

Mazloum, Abg Rita y Isea, Josia (2017). La flexibilidad en los horarios de trabajo como herramienta para mejorar la productividad del talento. lustitia Socialis. Revista Arbitrada de Ciencias Jurídicas. Año II. Vol. II. №3. julio - diciembre, 2017. Disponible en: https://www.academia.edu/37383868/. [En línea]. Fecha de Consulta: 24 de junio de 2018.

Organización Internacional del Trabajo (2001). La incidencia en el empleo de las fusiones y adquisiciones en el sector de los servicios financieros y de la banca. Publicación del Organización Internacional del Trabajo. Disponible en: http://ilo.org/global/publications/ilobooks_PUBL_9223123275_ES/. [En línea]. Fecha de Consulta: 25 de julio de 2019.

Quiroz, Antonio (2017). ¿Por qué es necesaria la flexibilidad laboral? Disponible en: https://www. wasabijobs.com/necesaria-la-flexibilidad-laboral/. [En línea]. Fecha de Consulta: 25 de junio de 2019.

Rimbau Gilabert, Eva y Triadó, Xavier (2006). La Flexibilidad Laboral en la Empresa. Revista Capital Humano, No. 197. Universitat Oberta de Catalunya y Universidad de Barcelona. Barcelona, España. Disponible en: https://www.researchgate.net/publication/304244765 La flexibilidad laboral en la empresa. [En Línea]. Fecha de Consulta: 23 de junio de 2018.

Vigo, Alejandro. (2002). Hans-Georg Gadamer y la filosofía hermenéutica: la comprensión como ideal y tarea. Revista de Estudios Públicos (87), pp. 235-249. Centro de Estudios Públicos, Santiago, Chile. Disponible en: http://www.cepchile.cl /archivo_1043/rev87_vigo.pdf. [En línea]. Fecha de consulta: 28 de junio de 2019.

Weber, M. (1996). Economía y sociedad. Fondo de Cultura Económica. México, D.F.

Weller, Jürgen (2009). Políticas para el perfeccionamiento de la institucionalidad laboral en Panamá. Comisión Económica para América Latina y el Caribe (CEPAL). Disponible en: https://core.ac.uk/download/pdf/38671949.pdf. [En línea]. Fecha de Consulta: 28 de julio de 2019. 